\title{
Priori-Information Aided Iterative Hard Threshold: A Low-Complexity High-Accuracy Compressive Sensing Based Channel Estimation for TDS-OFDM
}

\author{
Zhen Gao, Chao Zhang, Member, IEEE, Zhaocheng Wang, Senior Member, IEEE, Sheng Chen, Fellow, IEEE
}

\begin{abstract}
This paper develops a low-complexity channel estimation (CE) scheme based on compressive sensing (CS) for time-domain synchronous (TDS) orthogonal frequency-division multiplexing (OFDM) to overcome the performance loss under doubly selective fading channels. Specifically, an overlap-add method of the time-domain training sequence is first proposed to obtain the coarse estimates of the channel length, path delays and path gains of the wireless channel, by exploiting the channel's temporal correlation to improve the robustness of the coarse CE under the severe fading channel with long delay spread. We then propose the priori-information aided (PA) iterative hard threshold (IHT) algorithm, which utilizes the priori information of the acquired coarse estimate for the wireless channel and therefore is capable of obtaining an accurate channel estimate of the doubly selective fading channel. Compared with the classical IHT algorithm whose convergence requires the $l_{2}$ norm of the measurement matrix being less than 1, the proposed PA-IHT algorithm exploits the priori information acquired to remove such a limitation as well as to reduce the number of required iterations. Compared with the existing CS based CE method for TDSOFDM, the proposed PA-IHT algorithm significantly reduces the computational complexity of $\mathrm{CE}$ as well as enhances the $\mathrm{CE}$ accuracy. Simulation results demonstrate that, without sacrificing spectral efficiency and changing the current TDS-OFDM signal structure, the proposed scheme performs better than the existing CE schemes for TDS-OFDM in various scenarios, especially under severely doubly selective fading channels.
\end{abstract}

Index Terms-Orthogonal frequency-division multiplexing (OFDM), digital terrestrial television broadcasting, time-domain synchronous OFDM, compressive sensing, channel estimation

\section{INTRODUCTION}

Orthogonal frequency-division multiplexing (OFDM) technology has been widely applied in high-speed broadband wireless communication systems [1]. In the digital terrestrial television broadcasting (DTTB) field, both European second generation digital video broadcasting standard (DVB-T2) [2] and Chinese digital terrestrial multimedia broadcasting standard (DTMB) [3] adopt OFDM as the key modulation technology. DVB-T2 uses cyclic prefix (CP) based OFDM, where a $\mathrm{CP}$,

Z. Gao, C. Zhang, and Z. Wang are with Tsinghua National Laboratory for Information Science and Technology (TNList), Department of Electronic Engineering, Tsinghua University, Beijing 100084, China (E-mails: gao-z11@mails.tsinghua.edu.cn; z_c@tsinghua.edu.cn; zcwang@mail.tsinghua.edu.cn).

S. Chen is with Electronics and Computer Science, University of Southampton, Southampton SO17 1BJ, U.K. (E-mail: sqc@ecs.soton.ac.uk), and also with King Abdulaziz University, Jeddah 21589, Saudi Arabia.

This work was supported by National Key Basic Research Program of China (Grant No. 2013CB329203), National Nature Science Foundation of China (Grant No. 61271266), National High Technology Research and Development Program of China (Grant No. 2014AA01A704), and Beijing Natural Science Foundation (Grant No. 4142027). serving as the guard interval, is inserted between successive OFDM data blocks to eliminate the inter-block-interference (IBI) caused by multipath channels [4]. Unlike DVB-T2, DTMB uses time-domain synchronous (TDS) OFDM, which replaces the $\mathrm{CP}$ with a time-domain training sequence (TS). Compared with the classical CP based OFDM, TDS-OFDM has superior performance in terms of fast synchronization and channel estimation (CE), and it also achieves a higher spectral efficiency [5]-[7]. Owing to the good performance of TDSOFDM, DTMB has been officially approved as an international DTTB standard, and has been successfully deployed in China and several other countries [5].

However, because of the mutual interferences between the TS and the OFDM data block, an iterative interference cancellation has to be used in TDS-OFDM systems to decouple the $\mathrm{TS}$ and the OFDM data block for CE and frequency-domain demodulation [6]. This iterative interference cancellation will result in the performance degradation under doubly selective fading channels [5], whereby the perfect removal of the IBIs is difficult to realize. To solve this problem, several schemes have been proposed [7]-[9]. Among these solutions, the dual pseudo-noise OFDM (DPN-OFDM) has attracted more attention owing to its simplicity and its ability to deliver an accurate channel estimate without imposing complex iterative interference cancellation [7], at the cost of sacrificing some spectral efficiency. Recently, a channel estimation method was proposed based on compressive sensing (CS) for the current DTMB system [10], whereby the small IBI-free region in the received TS is exploited to estimate the long multipath channel by exploiting the greedy signal recovery algorithms for CS, such as the subspace pursuit (SP) algorithm [11] and the compressive sampling matching pursuit (CoSaMP) algorithm [12]. However, this scheme suffers from the drawback of high computational complexity owing to the matrix inversion operation required as well as the inevitable performance degradation under the adverse conditions of severe multipath channels with very long delay spread.

Against this background, in this paper we develop a lowcomplexity high-accuracy CS based CE scheme for TDSOFDM systems. Our contribution is twofold. Firstly, we derive the overlap-add method of the TS to obtain the coarse estimates of the channel length, path delays and path gains of the wireless channel, whereby the temporal correlation of the multipath delays and multipath gains among several consecutive TDS-OFDM symbols is exploited. More specifically, the TS tail part caused by the multipath channel is superposed on the preceding TS main part, and then this overlap-add result 
is circularly correlated with the local PN sequence to obtain some coarse channel state information (CSI). Moreover, the temporal correlation of the multipath delays and gains among several consecutive TDS-OFDM symbols is jointly exploited to improve the robustness and accuracy of the coarse CE. Assisted by the prior information of the wireless channel obtained by the overlap-add method, our proposed CS based $\mathrm{CE}$ method, referred to as the priori-information aided iterative hard threshold (PA-IHT), is capable of obtaining an accurate channel estimate while only imposing a low computational complexity. In particular, unlike the classical iterative hard threshold (IHT) algorithm [13] whose convergence requires the $l_{2}$ norm of the measurement matrix being less than 1 , our PA-IHT algorithm utilises the available priori information of the wireless channel to remove such a restriction as well as to reduce the number of required iterations. Also benefiting from the acquired priori information of the wireless channel, our PA-IHT algorithm significantly improves signal recovery accuracy as well as considerably reduces the computational complexity, in comparison to the the existing CS based CE method, such as the modified CoSaMP algorithm of [10]. Moreover, unlike the modified CoSaMP algorithm which suffers from invertible CE performance degradation under severe multipath channels with very long delay spread, the PA-IHT algorithm remains robust and accurate under such adverse channel conditions.

The rest of the paper is organised as follows. Section $\amalg$ presents the TDS-OFDM system model and briefly reviews several existing CE schemes for TDS-OFDM. Section III details our proposed PA-IHT based CE scheme for TDSOFDM, while Section IV provides the simulation results to demonstrate the superior performance of our PA-IHT algorithm over the existing state-of-the-arts. Our conclusions are drawn in Section $\mathrm{V}$.

Throughout our discussions, boldface capital and lower-case letters stand for matrices and column vectors, respectively. The exception is for frequency-domain vectors, whereby the discrete Fourier transform (DFT) of the time-domain vector $\mathbf{x}$ is denoted by $\boldsymbol{X}$. The operators $*$ and $\otimes$ represent the linear convolution and circular correlation, respectively, while $\lfloor\cdot\rfloor$ denotes the integer floor operator and $\operatorname{abs}\{\mathbf{x}\}$ is the vector whose elements are the absolute values of the corresponding elements of the vector $\mathbf{x}$. The transpose and conjugate transpose operations are denoted by $(\cdot)^{\mathrm{T}}$ and $(\cdot)^{\mathrm{H}}$, respectively, while $(\cdot)^{\dagger}$ denotes the Moore-Penrose matrix inversion and the $l_{p}$ norm operation is given by $\|\cdot\|_{p}$. The $r$-sparse vector of $\mathbf{x}$ is denoted by $\mathbf{x}\rangle_{r}$ which is generated by retaining the $r$ largest elements of $\mathbf{x}$ and setting the rest of the elements to zero. The support of the vector $\mathbf{x}$ is denoted by $\operatorname{supt}\{\mathbf{x}\}$, and $\left.\mathbf{x}\right|_{\Gamma}$ denotes the entries of $\mathbf{x}$ defined in the set $\Gamma$, while $\left.\boldsymbol{\Phi}\right|_{\Gamma}$ denotes the sub-matrix whose columns comprise the columns of $\boldsymbol{\Phi}$ that are defined in the set $\Gamma$. Additionally, $|\cdot|$ denotes the absolute value, and $|\cdot|_{c}$ is the cardinality of a set. Finally, $\delta(\cdot)$ represents the unit impulse function.

\section{SySTEM MODEL}

In the time domain, TDS-OFDM signals are grouped in symbols, and each TDS-OFDM symbol consists of a
TS, which is a known pseudo-noise $(\mathrm{PN})$ sequence $\mathbf{c}=$

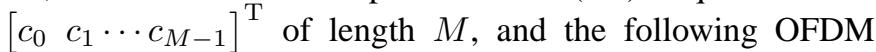
data block of length $N$, which can be expressed as $\mathbf{x}_{i}=$ $\left[\begin{array}{ll}x_{i, 0} & x_{i, 1} \cdots x_{i, N-1}\end{array}\right]^{\mathrm{T}}$ with $i$ denoting the TDS-OFDM symbol index. Hence, the $i$ th TDS-OFDM symbol is represented by $\mathbf{s}_{i}=\left[\mathbf{c}^{\mathrm{T}} \mathbf{x}_{i}^{\mathrm{T}}\right]^{\mathrm{T}}=\left[\mathbf{c}^{\mathrm{T}}\left(\mathbf{F}_{N}^{\mathrm{H}} \boldsymbol{X}_{i}\right)^{\mathrm{T}}\right]^{\mathrm{T}}$, where $\mathbf{F}_{N}$ is the DFT matrix of size $N \times N$ and $\boldsymbol{X}_{i}=\left[\begin{array}{lll}X_{i, 0} & X_{i, 1} \cdots X_{i, N-1}\end{array}\right]^{\mathrm{T}}$ is the frequency-domain $i$ th OFDM data block.

At the receiver, the received $i$ th OFDM symbol can be written as $\mathbf{r}_{i}=\mathbf{s}_{i} * \mathbf{h}_{i}+\mathbf{n}_{i}$, where $\mathbf{n}_{i}$ is the channel additive white Gaussian noise (AWGN) vector having a zero mean, while $\mathbf{h}_{i}=\left[h_{i, 0} h_{i, 1} \cdots h_{i, L-1}\right]^{\mathrm{T}}$ is the time-varying channel impulse response (CIR) of length $L$ which can be considered to be quasi-static during the time period of the $i$ th TDS-OFDM symbol. Since the wireless channel is sparse in nature [14], its CIR comprises only $P$ resolvable propagation paths, where $P \ll L$. In other words, only $P$ coefficients of $\mathbf{h}_{i}$ are non zero, and therefore the coefficients of the CIR can be expressed by the following model [15], [16]

$$
h_{i, l}=\sum_{p=0}^{P-1} \alpha_{i, p} \delta\left(l-\tau_{i, p}\right), 0 \leq l \leq L-1,
$$

where $\alpha_{i, p}$ is the $p$ th path gain and $\tau_{i, p}$ is the $p$ th path delay. Obviously, we have

$$
h_{i, l}=\left\{\begin{array}{cc}
\alpha_{i, p}, & \text { if } l=\tau_{i, p}, \\
0, & \text { otherwise. }
\end{array}\right.
$$

For CE and data demodulation in TDS-OFDM, the existing schemes may not be able to achieve satisfactory performance, especially under severe multipath channels. Fig. 1 illustrates several existing CE schemes for TDS-OFDM systems. As shown in Fig. 1 (a), the conventional CE scheme for TDSOFDM using single PN sequence has the advantage of maintaining a high spectral efficiency. However, this scheme suffers from the mutual interferences between the PN sequence and the OFDM data block. Therefore, an iterative interference cancellation has to be adopted to decouple the mutual interferences [6], which reduces the accuracy of CE in the doubly selective fading channel [5]. In the DPN-OFDM scheme, as shown in Fig. 1 (b), an extra PN sequence is inserted to prevent the second PN sequence from being contaminated

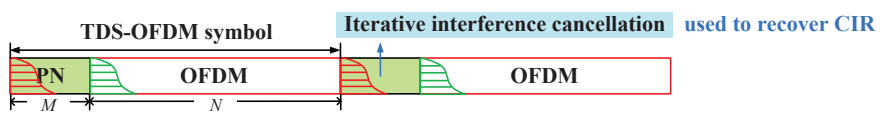

(a)

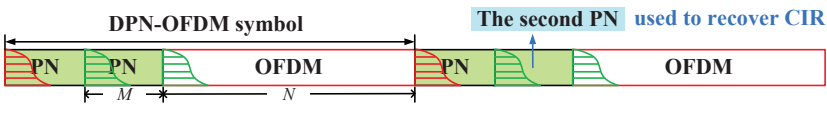

(b)

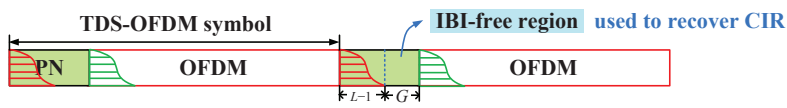

Fig. 1. Existing channel estimation schemes for TDS-OFDM: (a) the scheme based on iterative interference cancellation, (b) the scheme based on dual PN sequences, and (c) the scheme based on compressive sensing. 


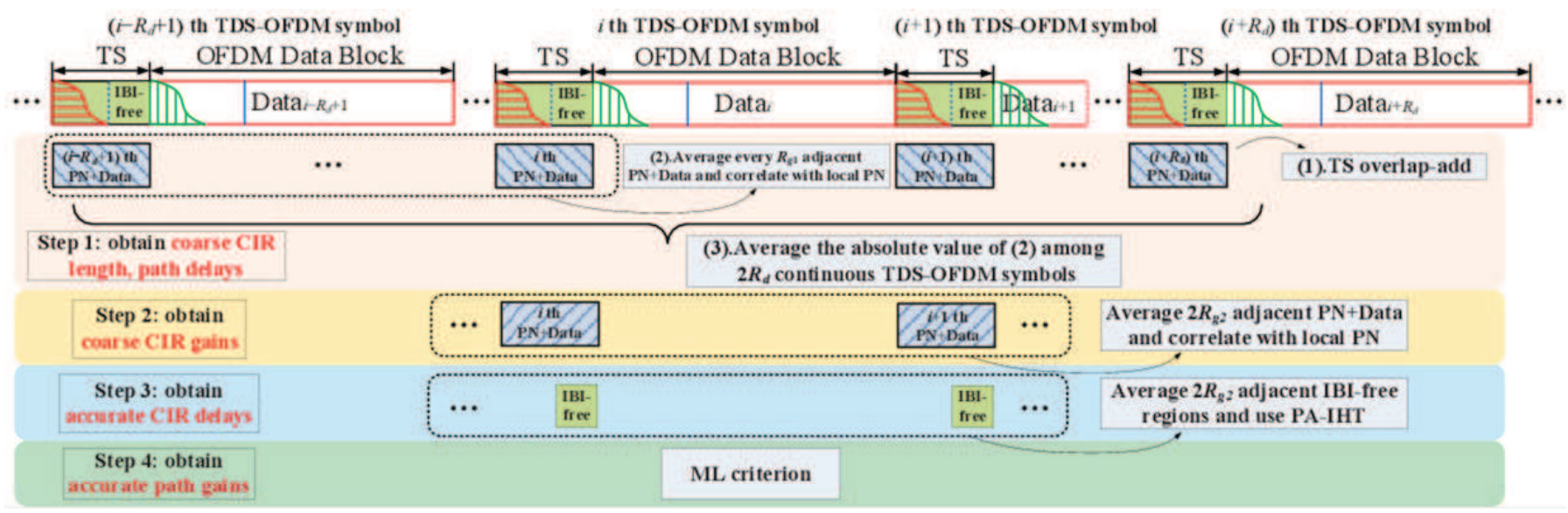

Fig. 2. The proposed PA-IHT based channel estimation which consists of four steps. The first two steps use the proposed overlap-add method of the TS, whereby the temporal correlation of the wireless channel is exploited to obtain some priori information of the wireless channel. In the rest two steps, the proposed PA-IHT algorithm and the ML criterion are used to obtain an accurate channel estimation.

by the preceding OFDM data block. In this way, the DPNOFDM scheme removes the complex iterative interference cancellation and improves the CE performance, but at the cost of reducing the spectral efficiency.

The PN sequence length in a TDS-OFDM system is designed to be longer than the maximum CIR length in order to ensure the reliable system performance. Considering the wireless scenarios that the actual CIR length $L$ is less or even much less than the length of the guard interval $M$, there is an IBI-free region of the small size $G=M-L+1$ at the end of the received PN sequence, as illustrated in Fig. 1 (c). In this IBI-free region, the received signal $\mathbf{y}=\left[\begin{array}{llll}y_{L-1} & y_{L} & \cdots & y_{M-1}\end{array}\right]^{\mathrm{T}}$ can be represented by

$$
\mathbf{y}=\mathbf{\Phi} \mathbf{h}+\mathbf{n}^{\prime},
$$

where $\mathbf{n}^{\prime}$ is the related channel AWGN vector, and

$$
\boldsymbol{\Phi}=\left[\begin{array}{cccc}
c_{L-1} & c_{L-2} & \cdots & c_{0} \\
c_{L} & c_{L-1} & \cdots & c_{1} \\
\vdots & \vdots & \vdots & \vdots \\
c_{M-1} & c_{M-2} & \cdots & c_{M-L}
\end{array}\right]_{G \times L}
$$

is a Toeplitz matrix of size $G \times L$ which is completely determined by the TS.

Generally speaking, the IBI-free region, as shown in Fig. 1 (c), is usually small. Thus it is difficult to obtain a unique solution to the unknown channel $\mathbf{h}$ in (3), since the observation dimension $G$ is usually smaller than the CIR dimension $L$. Fortunately, the compressive sensing theory [12] has proved that the high dimension signal can be accurately reconstructed by the low dimensional uncorrelated observations if the target signal is sparse or approximately sparse. A wireless channel is sparse in nature [14] and the actual number of the resolvable paths $P$ usually satisfies $P \ll L$. Therefore, even though the CIR dimension $L$ is larger or even much larger than the observation dimension $G$, we may have $P \leq G$. In [10], a modified CoSaMP algorithm is proposed to solve the under-determined equation (3). This scheme inherits the advantage of high spectral efficiency without changing the current TDS-OFDM signal structure. Furthermore, it improves the CE performance without the need of iterative interference cancellation. However, this scheme has a high computational complexity due to the required matrix inversion operations in the CS algorithm and moreover its performance degrades over severe multipath channels with long delay spread, where the observation dimension $G$ may become too small.

\section{PA-IHT Based Channel Estimation}

In this section, we present the CE method based on the proposed PA-IHT algorithm for TDS-OFDM systems and also provide the complexity analysis for this CE scheme.

\section{A. The Proposed PA-IHT Based Channel Estimation Method}

The proposed CE method consists of four steps, as shown in Fig. 2. To be more specific, in the first step, a coarse CIR length and path delays are estimated, while in the second step, coarse channel gains are obtained. With the aid of the coarse information of the wireless channel obtained in the first two steps, the proposed PA-IHT algorithm estimates the accurate path delays in the third step, and finally at the fourth step, the accurate path gains are obtained based on a maximum likelihood (ML) criterion.

In the first two steps, we exploit the temporal correlation of the wireless channel to estimate the coarse CIR length, path delays, and path gains. For time-varying channels, the path delays usually vary more slowly than the path gains [17]. Even for mobile scenarios, although the path gains will change over adjacent TDS-OFDM symbols, the path delays may remain relatively unchanged. This is because the duration $T_{\text {delay }}$ for the delay of a particular path to change by one tap is inversely proportional to the signal bandwidth $f_{s}$, while the coherence time of the path gains $T_{\text {gain }}$ is inversely proportional to the carrier frequency $f_{c}$ [17], [18]. Since $f_{s} \ll f_{c}$ for all practical wireless systems, path delays change much slower than path gains, i.e. $T_{\text {gain }} \ll T_{\text {delay }}$. Fig. 3 depicts the CIRs of four adjacent TDS-OFDM symbols over International Telecommunications Union Vehicular B (ITU-VB) channel [19] with $120 \mathrm{~km} / \mathrm{h}$ receiver velocity. From Fig. 3, we observe that although the path gains are different in adjacent TDSOFDM symbols, the path delays remain nearly invariant. The 
characteristics of the time-varying channel over several TDSOFDM symbols can therefore be exploited to assist the coarse CE.

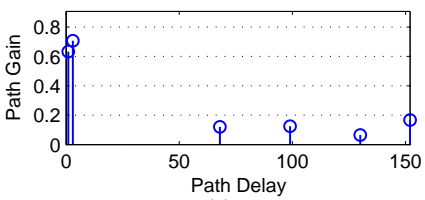

(a)

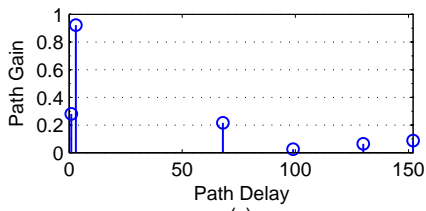

(c)
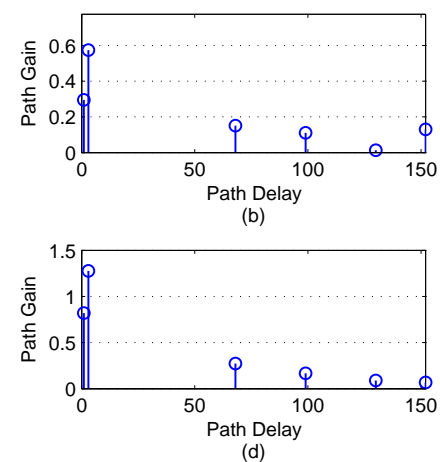

(d)
Fig. 3. The CIRs of four adjacent TDS-OFDM symbols over the ITUVB channel with $120 \mathrm{~km} / \mathrm{h}$ receiver velocity. The DTTB carrier frequency $f_{c}=634 \mathrm{MHz}$ and the symbol rate $1 / T_{s}=7.56 \mathrm{MHz}$.

Let us now elaborate this formally. Firstly, for a timevarying channel, the path delays of the channel in the time interval length of $T_{\text {delay }}$, or the duration of $\left|\frac{T_{\text {delay }}}{T_{s}(M+N)}\right|$ TDSOFDM symbols, can be considered to remain nearly unchanged [17], [18]. In other words, the CIR can be considered to share the same sparsity pattern during $2 R_{d}-1$ TDSOFDM symbols [20], where $R_{d}=\left\lfloor\frac{T_{\text {delay }}}{2 T_{s}(M+N)}\right\rfloor$, or in the time duration of $T_{\text {delay }}$ the temporally common sparsity of the wireless channel is guaranteed [18]. In practice, $R_{d}$ can be very large since $T_{\text {delay }} \propto \frac{T_{s} c}{v}=\frac{c}{f_{s} v}$, where $c$ is the velocity of light and $v$ is the speed of mobile receiver, while $T_{s}$ is the data symbol duration which may therefore be regarded as the resolution of delays. Hence we may obtain approximately $R_{d} \approx\left|\frac{c}{2 v(M+N) f_{s} T_{s}}\right|$. Taking the example of $v=100 \mathrm{~m} / \mathrm{s}$ and $M+N=256+4096$ for instance, $R_{d}$ can be considered to be unchanged during a superframe in the DTMB system, where a DTMB superframe consists of multiple TDS-OFDM symbols.

Secondly, over the coherence time of the path gains, $T_{\text {gain }}$, the channel gains can be expressed as $\left|\alpha_{i, p}\right| \exp \left(\phi_{0}+2 \pi f_{d} t\right)$, where $\phi_{0}$ is the initial phase, $t$ denotes the time, and $f_{d}$ is the Doppler frequency offset which can be estimated at the receiver [21]. Hence, the phase variation of a complex path gain is less than $\pi$ within the time interval of $\frac{1}{2 f_{d}}$ length, or the duration of $R_{g 1}=\left|\frac{1}{2 f_{d} T_{s}(M+N)}\right|$ TDS-OFDM symbols. In other words, the path delays of the channel are high correlated during $R_{g 1}=\left\lfloor\frac{1}{2 f_{d} T_{s}(M+N)}\right\rfloor$ TDS-OFDM symbols. By averaging the CIR estimate over $R_{g 1}$ adjacent TDS-OFDM symbols, therefore, we can reduce the effects of the channel AWGN and improve the accuracy and reliability of the path delay estimation. Clearly, for this averaging to be effective, we must have $R_{g 1}>1$.

It is well known that $f_{d} \propto \frac{f_{c} v}{c}$. Hence we may obtain approximately $R_{g 1} \approx\left\lfloor\frac{c}{2 v(M+N) f_{c} T_{s}}\right\rfloor$. Since $f_{s} \ll f_{c}$, clearly we have $2 R_{d}-1>R_{g 1}>1$. In practice, the receiver can adaptively choose appropriate values for the parameters $R_{d}$ and $R_{g 1}$ based on the channel status and the estimated $f_{d}$ of the time-varying channel.

Furthermore, in order to achieve the reliable coarse channel estimation in the case of instantaneous deep channel fading occurring during $R_{g 1}$ adjacent TDS-OFDM symbols, the CIR estimations over the $2 R_{d}-1$ adjacent TDS-OFDM symbols are jointly exploited to further improve the coarse estimation of the channel length and path delays.

Lastly, let us define the wireless channel being quasi-static during the duration of $2 R_{g 2}-1$ TDS-OFDM symbols. In general, we must assume that the path delays and path gains of the wireless channel remain unchanged at least during one TDS-OFDM symbol. Therefore, for time-varying channels, we can choose $R_{g 2}=1$.

For static channels in particular where $f_{d}=0$, both the path delays and path gains are time-invariant. We can simple choose a desired value of $R_{g 1}>1$ for averaging, and further set $2 R_{d}-1=2 R_{g 2}-1=R_{g 1}$. We now detail our proposed PA-IHT algorithm.

1) Step 1. Acquisition of Coarse Channel Length and Path Delays: We propose the overlap-add method of the TS, which jointly utilizes the received TSs from the $\left(i-R_{d}+1\right)$ th to $(i+$ $R_{d}$ )th TDS-OFDM symbols to exploit the temporal correlation of the wireless channel. The proposed overlap-add method of the TS is illustrated in Fig. 4, and its operation is represented by

$$
\mathbf{r}_{k}=\mathbf{r}_{k, \text { main }}+\mathbf{r}_{k, \text { tail }}, i-R_{d}+1 \leq k \leq i+R_{d}
$$

in which the TS main part $\mathbf{r}_{k \text {,main }}$ and the TS tail part $\mathbf{r}_{k \text {,tail }}$ can be expressed respectively by

$$
\begin{aligned}
\mathbf{r}_{k, \text { main }} & =\boldsymbol{\Psi}_{k} \mathbf{h}_{k}+\mathbf{n}_{k, \text { main }}, i-R_{d}+1 \leq k \leq i+R_{d}, \\
\mathbf{r}_{k, \text { tail }} & =\boldsymbol{\Theta}_{k} \mathbf{h}_{k}+\mathbf{n}_{k, \text { tail }}, i-R_{d}+1 \leq k \leq i+R_{d}
\end{aligned}
$$

where $\mathbf{n}_{k, \text { main }}$ and $\mathbf{n}_{k \text {,tail }}$ are the corresponding AWGN vectors, while

$$
\boldsymbol{\Psi}_{k}=\left[\begin{array}{ccccc}
c_{0} & x_{k-1, N-1} & x_{k-1, N-2} & \cdots & x_{k-1, N-L+1} \\
c_{1} & c_{0} & x_{k-1, N-1} & \cdots & x_{k-1, N-L+2} \\
\vdots & \vdots & \vdots & \ddots & \vdots \\
c_{L-1} & c_{L-2} & c_{L-3} & \cdots & c_{0} \\
\vdots & \vdots & \vdots & \ddots & \vdots \\
c_{M-1} & c_{M-2} & c_{M-3} & \cdots & c_{M-L}
\end{array}\right]_{M \times L}
$$

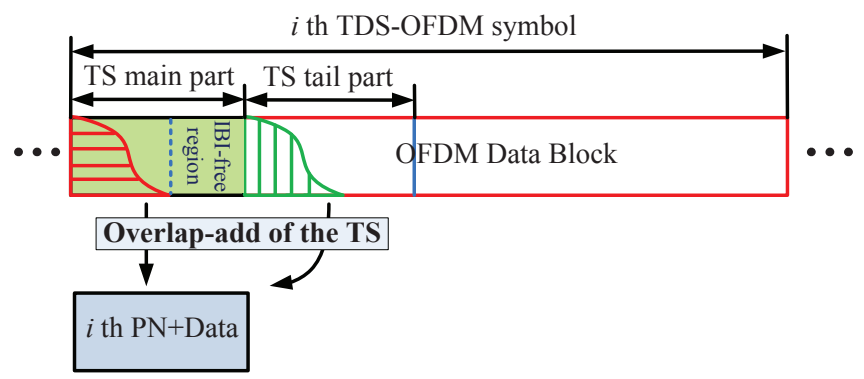

Fig. 4. Illustration for overlap-add of the TS in the $i$ th TDS-OFDM symbol. Note that in Step 1, the length of the TS tail part is $M$, while in Step 2, the length of the TS tail part is the estimated CIR length. 
$\boldsymbol{\Theta}_{k}=\left[\begin{array}{ccccc}x_{k, 0} & c_{M-1} & c_{M-2} & \cdots & c_{M-L+1} \\ x_{k, 1} & x_{k, 0} & c_{M-1} & \cdots & c_{M-L+2} \\ \vdots & \vdots & \vdots & \ddots & \vdots \\ x_{k, L-1} & x_{k, L-2} & x_{k, L-3} & \cdots & x_{k, 0} \\ \vdots & \vdots & \vdots & \ddots & \vdots \\ x_{k, M-1} & x_{k, M-2} & x_{k, M-3} & \cdots & x_{k, M-L}\end{array}\right]_{M \times L}$

Subsequently, the overlap-add results of the TS of $R_{g 1}$ adjacent TDS-OFDM symbols are averaged, and then circularly correlated with the known TS, whereby the good autocorrelation and circular cross-correlation property of the TS is exploited. Specifically, we have

$$
\begin{aligned}
\widetilde{\mathbf{h}}_{q}= & \frac{1}{M R_{g 1}}\left(\mathbf{c} \otimes \sum_{k=q}^{q+R_{g 1}-1} \mathbf{r}_{k}\right)=\frac{1}{R_{g 1}} \sum_{k=q}^{q+R_{g 1}-1} \mathbf{h}_{k}+\mathbf{v}_{k} \\
& i-R_{d}+1 \leq q \leq i+R_{d}-R_{g 1}
\end{aligned}
$$

where $\mathbf{v}_{k}$ represents the circular correlation of the interference plus the channel AWGN with the PN sequence averaging over the TS of $R_{g 1}$ adjacent TDS-OFDM symbols. Note that by averaging over $R_{g 1}$ adjacent TDS-OFDM symbols, the effect of the AWGN is significantly reduced. Consequently, the coarse $C E \overline{\mathbf{h}}$ is given by

$$
\overline{\mathbf{h}}=\frac{1}{2 R_{d}-R_{g 1}} \sum_{q=i-R_{d}+1}^{i+R_{d}-R_{g 1}} \operatorname{abs}\left\{\widetilde{\mathbf{h}}_{\mathrm{q}}\right\} .
$$

For time-varying channels, $2 R_{d}-R_{g 1}(>1)$ estimates $\mathbf{h}_{q}$ are utilized to obtain the coarse $\mathrm{CE} \hat{\mathbf{h}}$, and this reduces the effect of instantaneous deep channel fading occurring during the duration of a particular TDS-OFDM symbol. For static channels, $2 R_{d}-R_{g 1}=1$, and only single estimate $\mathbf{h}_{q}$ is used to obtain the coarse CE $\overline{\mathbf{h}}$, since the channel delays and gains are constant over adjacent TDS-OFDM symbols. Finally, only the propagation path delays of the most significant taps

$$
D_{0}=\left\{\tau_{1}:\left|\bar{h}_{\tau_{1}}\right| \geq E_{\mathrm{th}}\right\}_{\tau_{1}=0}^{L-1}
$$

are retained, and the retained $\left\{\bar{h}_{\tau_{1}}\right\}_{\tau_{1}=0}^{L-1}$ form the resulting coarse estimate $\overline{\mathbf{h}}$, where $E_{\mathrm{th}}$ is the power threshold which can be determined according to [22]. In this way, the channel length can be estimated from the coarse $\mathrm{CE}$ according to

$$
\widehat{L}=\max _{\tau_{1} \in D_{0}} \tau_{1}+a,
$$

where $a$ is a variable parameter used to define the IBI-free region comprising the last $G$ samples of the received TS, which can be determined according to [18].

With an initial channel sparsity level given by $S_{0}=\left|D_{0}\right|_{\mathrm{c}}$, the channel sparsity level is then determined according to $S=S_{0}+b$, where $b$ is a positive number used to combat the interference effect, since some low power paths may be treated as noise, and the value of $b$ can be calculated according to [18]. Effectively, $S$ is a coarse estimate of the number of resolvable propagation paths $P$.
2) Step 2. Acquisition of Coarse Channel Path Gains: The received TSs from $\left(i-R_{g 2}+1\right)$ th to $\left(i+R_{g 2}\right)$ th TDS-OFDM symbols are then used to provide the coarse estimate of the channel path gains according to

$$
\overline{\mathbf{h}}^{\prime}=\mathbf{c} \otimes \frac{1}{2 R_{g 2} M} \sum_{k=i-R_{g 2}+1}^{i+R_{g 2}}\left(\mathbf{r}_{k, \text { main }}+\mathbf{r}_{k, \text { tail }}^{\prime}\right)
$$

where $\mathbf{r}_{k \text {,tail }}^{\prime}$ is the vector whose first $\widehat{L}$ elements are the first $\widehat{L}$ of $\mathbf{r}_{k \text {,tail }}$, while its rest elements are all zeros.

The coarse estimates of the channel length, the channel path delays and path gains acquired in Steps 1 and 2 provide the priori information of the wireless channel to assist the accurate CE using the PA-IHT algorithm in the following two steps.

3) Step 3. Acquisition of Accurate Path Delay Estimate: We now present the proposed PA-IHT algorithm which utilizes the priori information from the coarse $\mathrm{CE}$ to improve the signal recovery accuracy and to reduce the computational complexity. Define the measurement vector as

$$
\overline{\mathbf{y}}=\frac{1}{2 R_{g 2}} \sum_{k=i-R_{g 2}+1}^{i+R_{g 2}} \mathbf{y}_{k},
$$

where $\mathbf{y}_{k}$ is the received signal vector in the IBI-free region of the $k$ th TDS-OFDM symbol as given in (3) but its size is $\widehat{G}=M-\widehat{L}+1$. The corresponding Toeplitz matrix $\mathbf{\Phi}$ of size $\widehat{G} \times \widehat{L}$ can be formed according to (4). The pseudocode of the proposed PA-IHT algorithm is summarized in Algorithm 1. The final estimated channel path delays are $D=\left\{\tau_{2}\right.$ : $\left.\left|\widehat{h}_{\tau_{2}}\right|>0\right\}_{\tau_{2}=0}^{L-1}$, with $\left\{\widehat{h}_{\tau_{2}}\right\}_{\tau_{2}=0}^{L-1}$ being the elements of $\widehat{\mathbf{h}}$.

Algorithm 1 Priori-Information Aided Iterative Hard Threshold (PA-IHT).

Input: 1) Initial path delay set $D_{0}$, coarse channel estimation $\overline{\mathbf{h}}^{\prime}$, channel sparsity level $S$;

2) Noisy measurements $\overline{\mathbf{y}}$, observation matrix $\boldsymbol{\Phi}$.

Output: $\mathrm{S}$-sparse estimation $\widehat{\mathbf{h}}$.

$$
\begin{aligned}
& \text { 1: }\left.\left.\mathbf{x}^{0}\right|_{D_{0}} \leftarrow \overline{\mathbf{h}}^{\prime}\right|_{D_{0}} \\
& \text { 2: } u_{\text {current }}=\left\|\overline{\mathbf{y}}-\mathbf{\Phi} \mathbf{x}^{0}\right\|_{2} \text {; } \\
& \text { 3: } u_{\text {previous }}=0 \text {; } \\
& \text { 4: while } u_{\text {previous }} \leq u_{\text {current }} \text {, do } \\
& k \leftarrow k+1 \text {; } \\
& \mathbf{z}=\mathbf{x}^{k-1}+\boldsymbol{\Phi}^{\mathrm{H}}\left(\overline{\mathbf{y}}-\boldsymbol{\Phi} \mathbf{x}^{k-1}\right) ; \\
& \left.\Gamma=\sup \{\operatorname{abs}\{\mathbf{z}\}\rangle_{\mathrm{S}}\right\} \text {; } \\
& \mathbf{x}^{k} \leftarrow \mathbf{x}^{k-1} \text {; } \\
& \left.\left.\mathbf{x}^{k}\right|_{\Gamma} \leftarrow \overline{\mathbf{h}}^{\prime}\right|_{\Gamma} \\
& \left.\mathbf{x}^{k} \leftarrow \mathbf{x}^{k}\right\rangle_{S} \\
& u_{\text {previous }}=u_{\text {current }} \text {; } \\
& u_{\text {current }}=\left\|\overline{\mathbf{y}}-\mathbf{\Phi} \mathbf{x}^{k}\right\|_{2} ; \\
& \text { 13: end while } \\
& \text { 14: } \widehat{\mathbf{h}} \leftarrow \mathbf{x}^{k-1} \text {. }
\end{aligned}
$$

In contrast to the classical IHT algorithm or other CS based algorithms, the proposed algorithm has several attractive features. Firstly, the PA-IHT algorithm exploits the available priori information of the coarse path delays and gains (or equivalently the locations and values of the partial large 
components in the target signal) as the initial condition, and this significantly enhances the signal recovery accuracy and reduces the number of required iterations. Secondly, unlike the modified CoSaMP algorithm [10], the sizes of the IBI-free region and the measurement matrix are adaptively determined by the coarse channel length estimate $\widehat{L}$. Thirdly, the coarse path gains serve as the nonzero element values of the target signal in every iteration. By contrast, in order to obtain these values, the modified CoSaMP algorithm has to apply the least squares estimation with high-complexity matrix inversion operation while the classical IHT algorithm uses the correlated results of the measurement matrix and the residual error [13], whose convergence condition requires that $\|\mathbf{\Phi}\|_{2}<1$.

4) Step 4. Accurate Path Gain Estimation Based on ML: The final channel estimate is obtained as the ML estimate [10]

$$
\left.\widehat{\mathbf{h}}^{\prime}\right|_{D}=\left(\left.\boldsymbol{\Phi}\right|_{D}\right)^{\dagger} \overline{\mathbf{y}}=\left(\left.\left(\left.\boldsymbol{\Phi}\right|_{D}\right)^{\mathrm{H}} \boldsymbol{\Phi}\right|_{D}\right)^{-1}\left(\left.\boldsymbol{\Phi}\right|_{D}\right)^{\mathrm{H}} \overline{\mathbf{y}},
$$

where $\widehat{\mathbf{h}}^{\prime}$ is a vector of length $M$, whose elements outside the set $D$ are zeros. We also give the Cramer-Rao lower bound (CRLB) of the proposed CE method [10]

$$
\operatorname{Var}_{\mathrm{CRLB}}=E\left\{\left\|\overline{\mathbf{h}}^{\prime}-\mathbf{h}\right\|_{2}\right\}=\frac{S}{2 R_{g 2} G \rho},
$$

where $\rho$ is the signal to noise ratio (SNR).

\section{B. Convergence Properties}

In contrast to the conventional greedy algorithms for CS [12] which are generally used to solve the problem of

$$
\min _{\mathbf{x}}\left\{\mathbf{x} \text { is an } S \text {-sparse vector : }\|\mathbf{y}-\mathbf{\Phi} \mathbf{x}\|_{2}\right\},
$$

the proposed PA-IHT algorithm in Step 3 is used for the support detection. This support detection can be written as

$$
\min _{D}\left\{D:\left\|\mathbf{y}-\left.\left.\mathbf{\Phi}\right|_{D} \overline{\mathbf{h}}^{\prime}\right|_{D}\right\|_{2}\right\}
$$

where $D$ is an $S$-dimension set, whose elements are in ascending order and they are specified by the indexes of the elements in $\overline{\mathbf{h}}^{\prime}$. Obviously, $D$ is uniquely determined when $\mathbf{y}, \boldsymbol{\Phi}$ and $\overline{\mathbf{h}}^{\prime}$ are given. The proposed algorithm solves the problem of (18) in a greedy manner. When the iterative procedure meets the stopping criterion, the algorithm converges to at least a locally optimal solution.

Moreover, from (14), it is clear that $\overline{\mathbf{h}}^{\prime}$ is an unbiased estimate of the channel, because the data part mixed in the overlap-add result of the TS can be regarded as a noise with zero mean. Thus the estimate of the coarse channel delays in Step 3 contains the true channel path delays with high probability [22]. By exploiting the priori information of the coarse channel path delays, the number of required iterations can be reduced, and the detected support tends to be a globally optimal solution.

\section{Computational Complexity}

Steps 1 and 2 implement the $M$-point circular correlation using fast Fourier transform (FFT), whose complexity is in the order of $\mathcal{O}\left(\left(M \log _{2} M\right) / 2\right)$. In Step 3, owing to the priori information of the acquired coarse channel gains, our algorithm avoids the matrix inversion operation. In Step 4, the ML estimate requires the matrix inversion operation with the complexity of $\mathcal{O}\left(G S^{2}+S^{3}\right)$. Obviously, the main computational burden comes from Step 4 . and the complexity of our proposed algorithm is $C_{\mathrm{PA}-\mathrm{IHT}}=\mathcal{O}\left(G S^{2}+S^{3}\right)$.

The conventional CoSaMP algorithm and the modified CoSaMP algorithm can be shown to have the computational complexity of $C_{\mathrm{CoSaMP}}=\mathcal{O}\left(4 G S^{3}+8 S^{4}\right)$ and $C_{\mathrm{mCoSaMP}}=$ $\mathcal{O}\left(\left(S-S_{0}\right)\left(4 G S^{2}+8 S^{3}\right)\right)$, respectively [10]. The main computational burden of those two algorithms comes from the matrix inversion operation required to obtain the sparsity information or nonzero element values in the target signal. By contrast, our algorithm acquires this information at the cost of very low complexity of $\mathcal{O}\left(\left(M \log _{2} M\right) / 2\right)$.

Considering the typical case of the ITU-VB channel [19] where we have $S=6, G=104$ and $S_{0}=3$. The computational complexity of the three schemes are given respectively by $C_{\mathrm{PA}-\mathrm{IHT}}=\mathcal{O}(3960), C_{\mathrm{CoSaMP}}=$ $\mathcal{O}(100224)$ and $C_{\mathrm{mCoSaMP}}=\mathcal{O}(50112)$. We then have $C_{\mathrm{PA}-\mathrm{IHT}} / C_{\mathrm{CoSaMP}} \approx 4 \%$ and $C_{\mathrm{PA}-\mathrm{IHT}} / C_{\mathrm{mCoSaMP}} \approx 8 \%$.

\section{Simulation Results}

A simulation study was carried out to compare the performance of the proposed PA-IHT scheme with those of the existing state-of-the-art methods for the TDS-OFDM system, including the modified CoSaMP based TDS-OFDM scheme [10] and the DPN-OFDM based scheme [7]. Simulation system parameters were set as: $f_{c}=643 \mathrm{MHz}, 1 / T_{s}=7.56 \mathrm{MHz}$, $N=2048$, and $M=256$ for the conventional TDSOFDM transmission and $M=2 \times 256$ for the DPN-OFDM transmission, while the perfect synchronization was assumed. We adopted the ITU-VB channel [19] and the China digital television test 8th channel model (CDT-8) channel [22] in the simulation, where both the static and mobile scenarios were investigated. The parameters $R_{g 1}$ and $R_{g 2}$ were adaptively set based on the channel state, while we considered $R_{d}=40$ in both the mobile and static scenarios. The simulation was carried out using MATLAB R2012a tool. In the simulation,

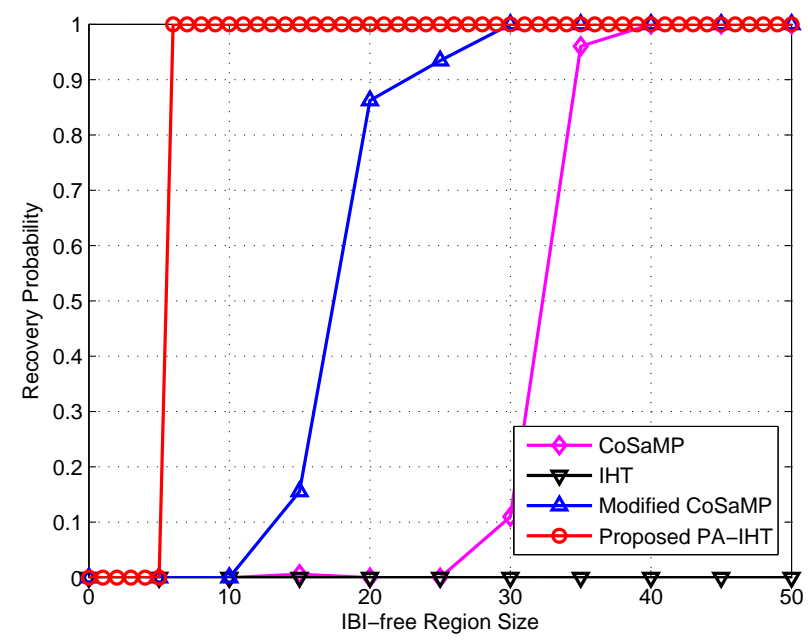

Fig. 5. Target signal recovery probabilities versus IBI-free region size attained by the four schemes for the static ITU-VB channel given $\mathrm{SNR}=20 \mathrm{~dB}$. 
the variable parameter $a$ in (13) was approximately chosen as $a \approx 0.1 \max _{\tau_{1} \in D_{0}} \tau_{1}$, while the positive number $b$ in determining the channel sparsity level $S$ was empirically set to $b \in[0,5]$ where the chosen value of $b$ was inversely related to SNR.

Fig. 5 shows the signal recovery probabilities as the function of the IBI-free region size $G$ achieved by the four different algorithms for the static ITU-VB channel, given SNR $=20 \mathrm{~dB}$. In this simulation, if the mean square error (MSE) of the signal estimation was lower than $10^{-2}$, the recovery result was considered to be correct [10] and hence the signal recovery probability was assumed to be 1 . It can be clearly seen from Fig. 5 that the proposed PA-IHT algorithm outperforms the other three algorithms significantly. The original IHT algorithm fails to work in this case, because its convergence requires that $\|\Phi\|_{2}<1[13]$, but the measurement matrix (4) does not meet this condition. Compared with the CoSaMP algorithm and the modified CoSaMP algorithm which require the IBI-free region of size 40 and 30, respectively, to recovery the signal correctly with probability 1 , the proposed PA-IHT algorithm only needs an IBI-free region of size 7. This means that the PA-IHT algorithm reduces the required observation samples by $82.5 \%$ and $76.7 \%$, respectively, compared with the CoSaMP algorithm and the modified CoSaMP algorithm. This is because the proposed algorithm benefits from the priori information acquired, in terms of both the locations and the values of the partial large components in the target signal. Therefore, our PA-IHT algorithm are particularly effective in combating the CIR with a very longer delay spread, while the existing CS based schemes may suffer from the serious performance degradation under such adverse channel conditions.

Fig. 6 compares the CIR estimates obtained by the three schemes for the time-varying CDT-8 channel with $120 \mathrm{~km} / \mathrm{h}$ receiver velocity given $\mathrm{SNR}=10 \mathrm{~dB}$. It can be clearly seen

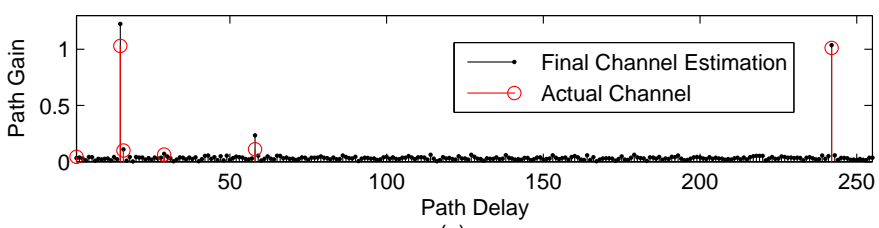

(a)

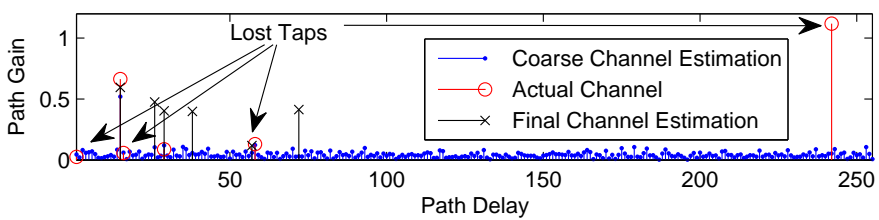

(b)

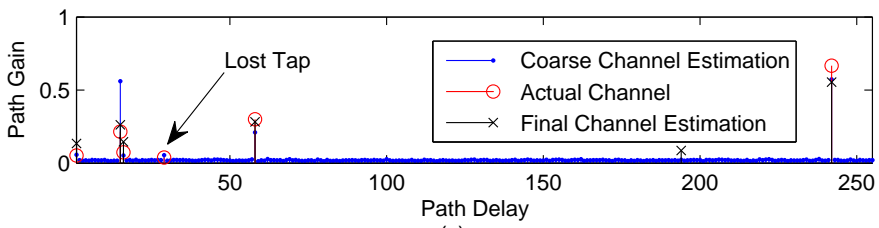

(c)

Fig. 6. Time-domain CIR estimates of the three different schemes for the CDT-8 channel with the mobile speed of $120 \mathrm{~km} / \mathrm{h}$ and given $\mathrm{SNR}=10 \mathrm{~dB}$ (a) the DPN-OFDM based scheme, (b) the modified CoSaMP based scheme, and (c) the proposed PA-IHT based scheme. from Fig. 6 (b) that the modified CoSaMP based scheme performs poorly. Four actual channel path taps, including the strongest echo path with a long delay spread, are missing from the CIR estimate provided by this scheme. By contrast, only one relatively insignificant channel path tap is missing from the CIR estimate obtained by the proposed scheme, as can be observed from Fig. 6 (c). This is because the CDT-8 channel has a very strong $0 \mathrm{~dB}$ echo with an extremely long delay spread. The coarse CE method in the modified CoSaMP scheme of [10] only uses the TS main part and discards the TS tail part (see the illustration of Fig. 4). Therefore, it cannot effectively detect the path delays with long delay spreads. By contrast, the proposed overlap-add method of the TS resolves this problem effectively. Moreover, by exploiting the temporal

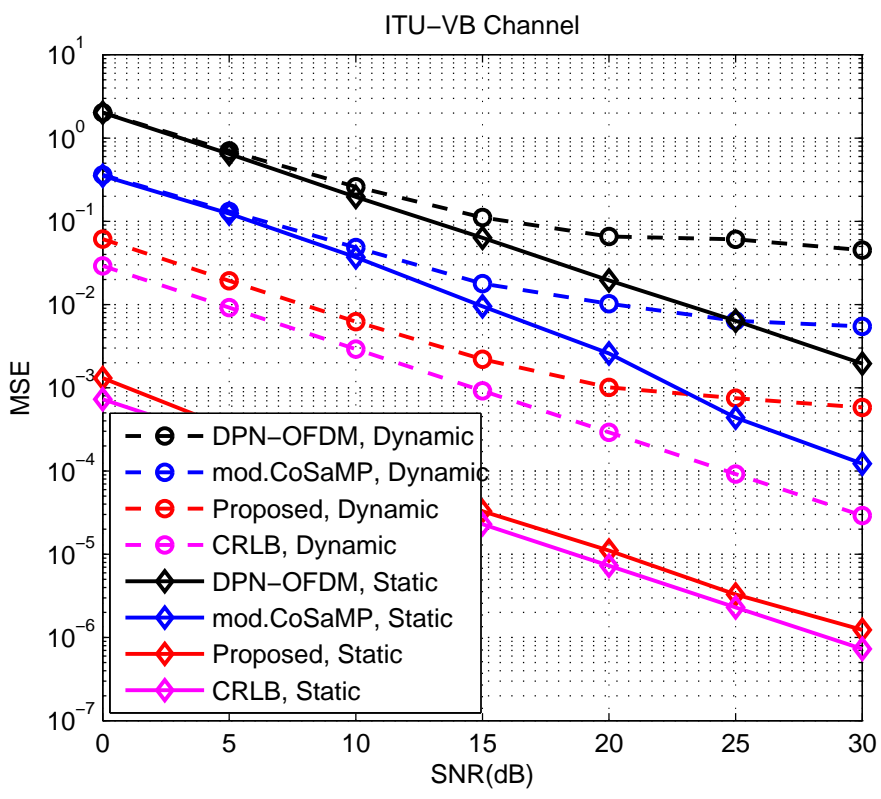

(a)

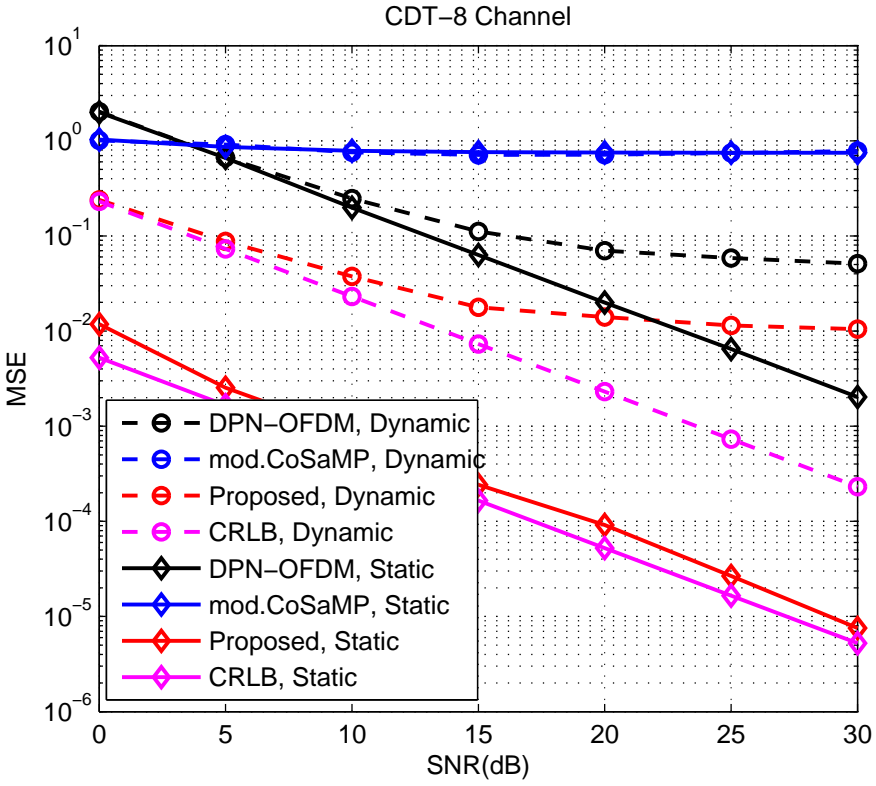

(b)

Fig. 7. MSE performance comparison of the proposed PA-IHT scheme with the existing DPN-OFDM based and modified CoSaMP based schemes: (a) the ITU-VB channel, and (b) the CDT-8 channel. 
correlation of the wireless channel, the proposed PA-IHT scheme significantly improves the robustness of the coarse CE. Since the modified CoSaMP scheme [10] only utilises the TSs preceding and following the current OFDM data block, its coarse path delay detection may fail to work properly under instantaneous deep fading channel situations. From Fig. 4 (a), it can be observed that the estimated gains of the first and fourth paths by the DPN-OFDM based scheme are lower than the noise floor. Hence, from the obtained CIR estimate, we cannot decide the delays of the first and fourth paths. Similar to the modified CoSaMP scheme, the DPN-OFDM based scheme suffers from serious performance degradation under instantaneous deep fading channel conditions as some of the estimated channel taps may be buried by the noise. Furthermore, the DPN-OFDM based scheme has an additional drawback of lower spectral efficiency.

Figs. 7 and 8 compare the achievable CE MSE performance and the data demodulation bit error rate (BER) performance of the three schemes, respectively, where the dynamic channel refers to the CDT- 8 or the ITU-VB channel with the mobile speed of $120 \mathrm{~km} / \mathrm{h}$. The modulation scheme employed was the quadrature phase shift keying (QPSK). It is clear that the existing modified CoSaMP based scheme achieves better performance than the existing DPN-OFDM based scheme for the ITU-VB channel, but the modified CoSaMP based method completely fails for the CDT-8 channel. This again confirms that the modified CoSaMP scheme suffers from serious performance degradation under severe multipath propagation environments. The results of Figs. 7 and 8 clearly demonstrate that our PA-IHT based scheme significantly outperforms the two existing schemes in various wireless scenarios, especially in fast time-varying and severe multipath propagation scenarios, such as the CDT- 8 channel with $120 \mathrm{~km} / \mathrm{h}$ receiver velocity. More specifically, for the static ITU-VB channel, the MSE performance of the proposed PA-IHT based scheme are more than $20 \mathrm{~dB}$ and $30 \mathrm{~dB}$ better than the modified CoSaMP and DPN-OFDM based schemes, respectively, while it outperforms the other two schemes by approximately $8 \mathrm{~dB}$ and $15 \mathrm{~dB}$, respectively, for the dynamic ITU-VB channel. For the dynamic and static CDT-8 channels, the MSE performance attained by our method are more than $5 \mathrm{~dB}$ and $20 \mathrm{~dB}$ better than the DPN-OFDM based method, respectively. Moreover, the MSE performance of our proposed method is very close to the theoretical CRLB for the two static channels. In terms of achievable BER, the superior performance of our scheme over the two existing ones are self-evident in Fig. 8, where the performance gain of our method over the existing methods is particularly noticeable under doubly selective fading channel environments. This is owing to the following reasons. The overlap-add method of the TS based on several consecutive TDS-OFDM symbols significantly improves the robustness and accuracy of the coarse estimates for the channel length and path delays. This provides the accurate priori information to assist the PA-IHT algorithm. Furthermore, the sizes of the IBIfree region and the measurement matrix are adaptive, which further improves the CE accuracy of the PA-IHT algorithm. It is also worth pointing out again that the proposed scheme does not alter the current TDS-OFDM signal structure and it

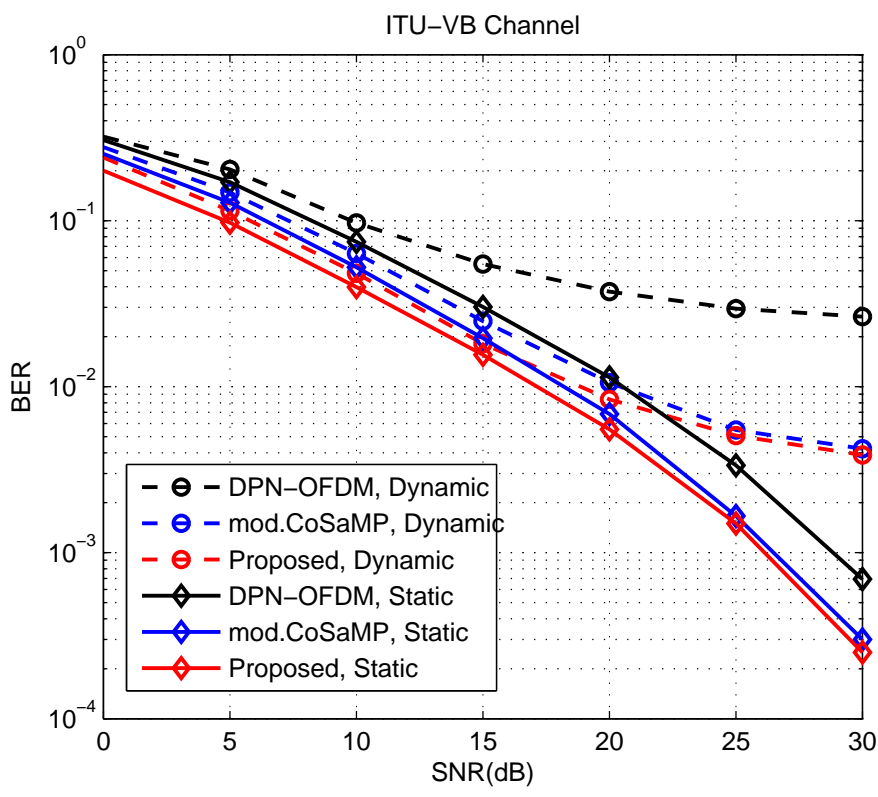

(a)

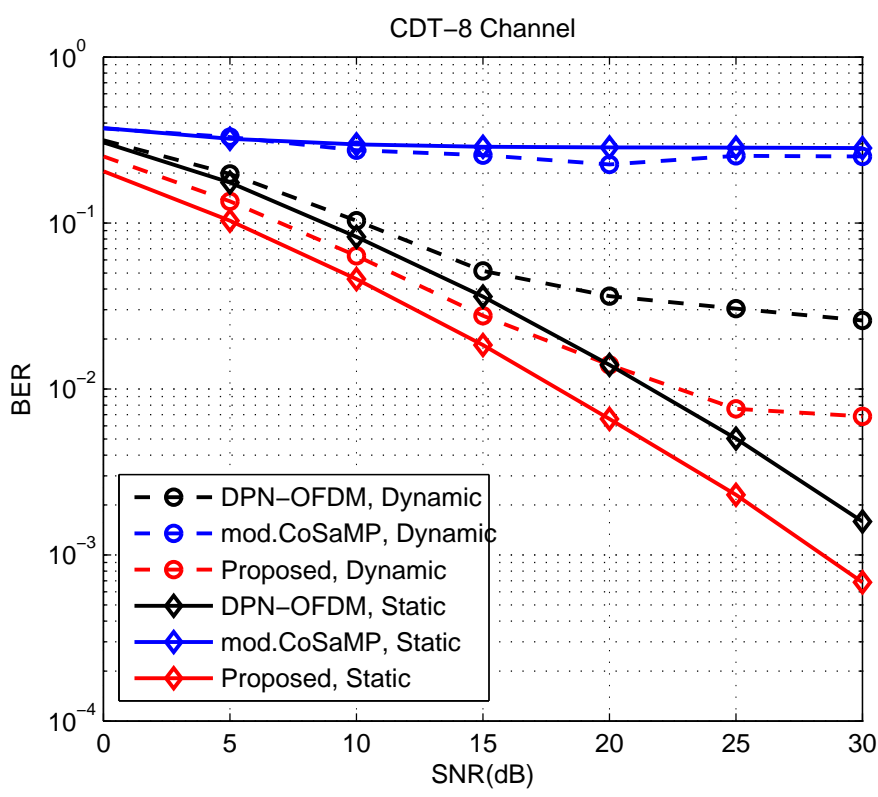

(b)

Fig. 8. BER performance comparison of the proposed PA-IHT scheme with the existing DPN-OFDM based and modified CoSaMP based schemes: (a) the ITU-VB channel, and (b) the CDT-8 channel. The modulation scheme employed is QPSK.

achieves a higher spectral efficiency than the existing DPNOFDM scheme.

\section{CONCLUSIONS}

In this paper, we have proposed a low-complexity and high-accuracy compressive sensing based channel estimation method, referred to as the PA-IHT algorithm, for widely deployed TDS-OFDM systems, which significantly outperforms the existing state-of-the-arts in terms of both estimation accuracy and computational complexity. The classical IHT algorithm for TDS-OFDM requires that the $l_{2}$ norm of the measurement matrix is smaller than 1 in order to guarantee convergence. By contrast, our proposed PA-IHT algorithm 
removes such a restriction and it only requires a very few iterations. We have also demonstrated that our scheme significantly outperforms the conventional DPN-OFDM based scheme. Compared with the DPN-OFDM scheme, our proposed PA-IHT based scheme has the additional advantage of achieving a higher spectral efficiency and it does not alter the current TDS-OFDM signal structure. Compared with the existing CS based methods, such as the modified CoSaMP algorithm for TDS-OFDM, our PA-IHT algorithm significantly improves the accuracy of the channel estimate while imposing a much lower computational complexity. Most significantly, our scheme maintains its effectiveness under fast time-varying severe multipath environments. Under such adverse channel conditions, the existing CS based scheme for TDS-OFDM fails to work completely.

\begin{tabular}{|c|c|}
\hline \multicolumn{2}{|c|}{ APPENDIX LIST OF ABBREVIATIONS } \\
\hline AWGN & Additive white Gaussian noise \\
\hline BER & Bit error rate \\
\hline CDT-8 & China digital television test 8 th channel model \\
\hline $\mathrm{CE}$ & Channel estimation \\
\hline CIR & Channel impulse response \\
\hline CoSaMP & Compressive sampling matching pursuit \\
\hline $\mathrm{CP}$ & Cyclic prefix \\
\hline CRLB & Cramer-Rao lower bound \\
\hline CS & Compressive sensing \\
\hline CSI & Channel state information \\
\hline DFT & Discrete Fourier transform \\
\hline DPN & Dual pseudo-noise \\
\hline DTMB & $\begin{array}{l}\text { Chinese digital terrestrial multimedia broad- } \\
\text { casting standard }\end{array}$ \\
\hline DTTB & Digital terrestrial television broadcasting \\
\hline DVB-T2 & $\begin{array}{l}\text { European second generation digital video } \\
\text { broadcasting standard }\end{array}$ \\
\hline FFT & Fast Fourier transform \\
\hline IBI & Inter-block-interference \\
\hline IHT & Iterative hard threshold \\
\hline ITU-VB & $\begin{array}{l}\text { International Telecommunications Union Ve- } \\
\text { hicular B }\end{array}$ \\
\hline ML & Maximum likelihood \\
\hline MSE & Mean square error \\
\hline OFDM & Orthogonal frequency-division multiplexing \\
\hline PA-IHT & $\begin{array}{l}\text { Priori-information aided iterative hard thresh- } \\
\text { old }\end{array}$ \\
\hline PN & Pseudo-noise \\
\hline QPSK & Quadrature phase shift keying \\
\hline SNR & Signal to noise ratio \\
\hline SP & Subspace pursuit \\
\hline TDS & Time-domain synchronous \\
\hline TS & Training sequence \\
\hline
\end{tabular}

\section{REFERENCES}

[1] J. A. C. Bingham, "Multicarrier modulation for data transmission: an idea whose time has come," IEEE Communications Magazine, vol. 28, no. 5, pp. 5-14, May 1990.

[2] Digital Video Broadcasting (DVB); Frame Structure, Channel Coding and Modulation for a Second Generation Digital Terrestrial Television Broadcasting System (DVB-T2). ETSI Standard, EN 302 755, V1.3.1, April 2012.
[3] Framing Structure, channel Coding and Modulation for Digital Television Terrestrial Broadcasting System. International DTTB Standard, GB 20600-2006, Aug. 2006.

[4] T. van Waterschoot, V. Le Nir, J. Duplicy, and M. Moonen, "Analytical expressions for the power spectral density of CP-OFDM and ZP-OFDM signals," IEEE Signal Processing Letters, vol. 17, no. 4, pp. 371-374, April 2010.

[5] L. Dai, Z. Wang, and Z. Yang, "Next-generation digital television terrestrial broadcasting systems: Key technologies and research trends," IEEE Communications Magazine, vol. 50, no. 6, pp. 150-158, June 2012.

[6] J. Wang, Z. Yang, C. Pan, and J. Song, "Iterative padding subtraction of the PN sequence for the TDS-OFDM over broadcast channels," IEEE Trans. Consumer Electronics, vol. 51, no. 4, pp. 1148-1152, Nov. 2005.

[7] J. Fu, J. Wang, J. Song, C. Pan, and Z. Yang, "A simplified equalization method for dual PN-sequence padding TDS-OFDM systems," IEEE Trans. Broadcasting, vol. 54, no. 4, pp. 825-830, Dec. 2008.

[8] M. Huemer, A. Onic, and C. Hofbauer, "Classical and Bayesian linear data estimators for unique word OFDM," IEEE Trans. Signal Processing, vol. 59, no. 12, pp. 6073-6085, Dec. 2011.

[9] L. Dai, Z. Wang, and Z. Yang, "Time-frequency training OFDM with high spectral efficiency and reliable performance in high speed environments," IEEE J. Selected Areas in Communications, vol. 30, no. 4, pp. 695-707, May 2012.

[10] L. Dai, Z. Wang, and Z. Yang, "Compressive sensing based timing domain synchronous OFDM transmission for vehicular communications," IEEE J. Selected Areas in Communications, vol. 31, no. 9, pp. 460-469, Sept. 2013.

[11] W. Dai and O. Milenkovic, "Subspace pursuit for compressive sensing signal reconstruction," IEEE Trans. Information Theory, vol. 55, no. 5 pp. 2230-2249, May 2009.

[12] M. F. Duarte and Y. C. Eldar, "Structured compressed sensing: from theory to applications," IEEE Trans. Signal Processing, vol. 59, no. 9 , pp. 4053-4085, Sept. 2011.

[13] T. Blumensath and M. E. Davies, "Iterative thresholding for sparse approximations," J. Fourier Analysis and Applications, vol. 14, nos. 5-6, pp. 629-654, Dec. 2008.

[14] W. U. Bajwa, J. Haupt, A. M. Sayeed, and R. Nowak, "Compressed channel sensing: a new approach to estimating sparse multipath channels," Proc. IEEE, vol. 98, no. 6, pp. 1058-1076, June 2010.

[15] B. Yang, K. B. Letaief, R. S. Cheng, and Z. Cao, "Channel estimation for OFDM transmission in multipath fading channels based on parametric channel modeling," IEEE Trans. Communications, vol. 49, no. 3, pp. 467-479, March 2001.

[16] A. Iyer, C. Rosenberg, and A. Karnik, "What is the right model for wireless channel interference?" IEEE Trans. Wireless Communications, vol. 8, no. 5, pp. 2662-2671, May 2009.

[17] I. E. Telatar and D. N. C. Tse, "Capacity and mutual information of wideband multipath fading channels," IEEE Trans. Information Theory, vol. 46, no. 4, pp. 1384-1400, July 2000.

[18] L. Dai, J. Wang, Z. Wang, P. Tsiaflakis, and M. Moonen, "Spectrum and energy-efficient OFDM based on simultaneous multi-channel reconstruction," IEEE Trans. Signal Processing, vol. 61, no. 23, pp. 6047-6059, Dec. 2013.

[19] C. Zhang, Z. Wang, C. Pan, S. Chen, and L. Hanzo, "Low-complexity iterative frequency domain decision feedback equalization," IEEE Trans. Vehicular Technology, vol. 60, no. 3, pp. 1295-1301, March 2011.

[20] E. van den Berg and M. P. Friedlander, "Theoretical and empirical results for recovery from multiple measurements," IEEE Trans. Information Theory, vol. 56, no. 5, pp. 2516-2527, May 2010.

[21] J. Cai, W. Song, and Z. Li, "Doppler spread estimation for mobile OFDM systems in Rayleigh fading channels," IEEE Trans. Consumer Electronics, vol. 49, no. 4, pp. 973-977, Nov. 2003.

[22] F. Wan, W.-P. Zhu, and M. N. S. Swamy, "Semi-blind most significant tap detection for sparse channel estimation of OFDM systems," IEEE Trans. Circuits and Systems, Part I, vol. 57, no. 3, pp. 703-713, March 2010 . 